\title{
Caracterización geoquímica de los depósitos alimentados por fuentes de lava del volcán Las Herrerías (Región Volcánica del Campo de Calatrava, Ciudad Real)
}

\author{
Geochemical characterization of the fire-fountain fed deposits \\ of Las Herrerías volcano (Campo de Calatrava Volcanic \\ Field, Ciudad Real)
}

\author{
F. Sarrionandia' 1 M. Carracedo Sánchez² ${ }^{2}$ L. Eguíluz¹, J.I. Gil Ibarguchi² \\ 1 Dpto. Geodinámica, Facultad de Farmacia, Universidad del País Vasco UPV/EHU, Paseo de las Universidades, 7, 01006 \\ Vitoria-Gasteiz (Álava). Email: fernando.sarrionandia@ehu.es; luis.eguiluz@ehu.es \\ 2 Dpto. Mineralogía y Petrología, Facultad de Ciencia y Tecnología, Universidad del País Vasco UPV/EHU, Sarriena s/n, \\ 48940 Leioa (Vizcaya). Email: manuel.carracedo@ehu.es; josei.gil@ehu.es
}

\begin{abstract}
RESUMEN
El volcán Las Herrerías (Bolaños de Calatrava; Región Volcánica del Campo de Calatrava) se caracteriza por una gran cantidad y variedad de depósitos originados a partir de fuentes de lava. Todos estos depósitos son composicionalmente idénticos, estando constituidos por melanefelinitas fuertemente sódicas $\left(\mathrm{Na}_{2} \mathrm{O}=2.24-3.81 \%\right)$, ricas en magnesio $(\mathrm{MgO}=11.58-14.19 \%)$, pobres en aluminio $\left(\mathrm{Al}_{2} \mathrm{O}_{3}=9.64-10.99 \%\right)$ y con elevados contenidos en tierras raras $\left(10 x-200 x\right.$ condrito), fundamentalmente más en tierras raras ligeras que en pesadas $\left[(\mathrm{La} / \mathrm{Lu})_{\mathrm{N}}=32-35\right]$. Los contenidos en $\mathrm{Ni}(233-286 \mathrm{ppm}), \mathrm{Cr}(393-520 \mathrm{ppm})$ y el número de magnesio $\left(\mathrm{Mg}^{*}=45-54\right)$ indican que no son magmas primarios, a diferencia de la mayor parte de las melanefelinitas equivalentes de esta región volcánica. Por otro lado, las ligeras variaciones composicionales mostradas por las muestras estudiadas responderían al efecto de la irregular distribución del clinopiroxeno en el magma durante la erupción. Los datos obtenidos son coherentes con el desarrollo de estas fuentes de lava en un ambiente de intraplaca continental.
\end{abstract}

Palabras clave: Geoquímica; spatter; melanefelinita; fuente de lava; Las Herrerías; Calatrava

\section{ABSTRACT}

The Las Herrerías volcano (Bolaños de Calatrava, Campo de Calatrava Volcanic Field) is characterized by the great amount and variety of fire-fountain fed deposits. All these deposits are compositionally similar, being constituted by magnesium-rich $(\mathrm{MgO}=11.58-4.19 \%)$, aluminium-poor $\left(\mathrm{Al}_{2} \mathrm{O}_{3}=9.64-10.99 \%\right)$ highly sodic $\left(\mathrm{Na}_{2} \mathrm{O}=2.24-3.81 \%\right)$ melanephelinites, with high contents in rare earth-elements $(10 \mathrm{x}-200 \mathrm{x}$ chondrite), particularly in light-rare earth elements with respect to the heavy ones $\left[(\mathrm{La} / \mathrm{Lu})_{\mathrm{N}}=32-35\right]$. Contrary to the equivalent melanephelinites of this volcanic field, the relatively low contents in Ni (233-286 ppm), Cr (393-520 ppm) and magnesium number $\left(\mathrm{Mg}^{*}=45-54\right)$ indicate that these rocks do not correspond with primary melts. On the other

Recibido el 28 de marzo de 2014; Aceptado el 16 de junio de 2014; Publicado el 16 de octubre de 2014

Citation / Cómo citar este artículo: F. Sarrionandia. et al. (2014). Caracterización geoquímica de los depósitos alimentados por fuentes de lava del volcán Las Herrerías (Región Volcánica del Campo de Calatrava, Ciudad Real). Estudios Geológicos, $70(2)$ : e012. http://dx.doi.org/10.3989/egeol.41726.317.

Copyright: () 2014 CSIC. This is an open-access article distributed under the terms of the Creative Commons Attribution-Non Commercial (by-nc) Spain 3.0 License. 
hand, the variable distribution of clinopyroxene in the magma during eruption would be responsible for the slight compositional differences observed in the studied samples. Finally, we argue that these fire fountains were developed in a continental intraplate setting.

Keywords: Geochemistry; spatter; melanephelinite; fire-fountain; Las Herrerías; Calatrava

\section{Introducción}

El volcán Las Herrerías está situado en el extremo oriental de la Región Volcánica del Campo de Calatrava (RVCC), a escasos $2 \mathrm{~km}$ al sureste de la localidad de Bolaños de Calatrava (Ciudad Real, España; Fig. 1).

La RVCC (e.g. Ancochea, 2004 y referencias incluidas allí) está integrada por más de 200 volcanes repartidos en un área de unos $5.000 \mathrm{~km}^{2}$, mayoritariamente dentro de la provincia de Ciudad Real. Estos edificios presentaron una actividad eminentemente efusiva y explosiva hace unos $8.7 \mathrm{Ma}$ (e.g. Ancochea, 2004 y referencias incluidas), actividad que quizás, aunque a falta de mayores comprobaciones, pudo haberse extendido hasta edades mucho más recientes (6500 años; González et al., 2007). Las rocas volcánicas generadas en estas erupciones son básicas - ultrabásicas y fuertemente alcalinas: leucititas, nefelinitas, melilititas y basaltos alcalinos (Ancochea, 1982; Cebriá, 1992).

Los productos y las morfologías volcánicas preservados en la RVCC indican que los estilos eruptivos dominantes fueron de tipo estromboliano e hidromagmático, es por ello que los maares y conos de escorias son los edificios volcánicos más abundantes. Las coladas de lava también son volumétricamente importantes, y la mayoría parten de conos de escoria.

Hasta hace unos años no se habían encontrado depósitos volcánicos que pudieran relacionarse claramente con las fuentes de lava típicas de las erupciones explosivas de estilo hawaiano (e.g. Stovall et al., 2010). Tales fuentes de lava lanzan al aire piroclastos muy fluidos y calientes (de hasta 1-2 m de diámetro) que son emitidos desde el cráter a velocidades de $\approx 100 \mathrm{~m} / \mathrm{s}$ y habitualmente ascienden hasta alturas de unas pocas decenas a unos pocos cientos de metros sobre la boca eruptiva antes de caer al suelo (e.g. 30-580 m; erupción en 1959 del volcán Kilauea Iki; Stovall et al. 2010). Los piroclastos emitidos en la parte central de la fuente de lava llegan al suelo muy fluidos y con altas temperaturas, y se acumulan muy rápido, pudiendo coalescer y generar lagos de lava o coladas de lava. Por otro lado, los piroclastos emitidos en la parte externa de la fuente de lava llegan

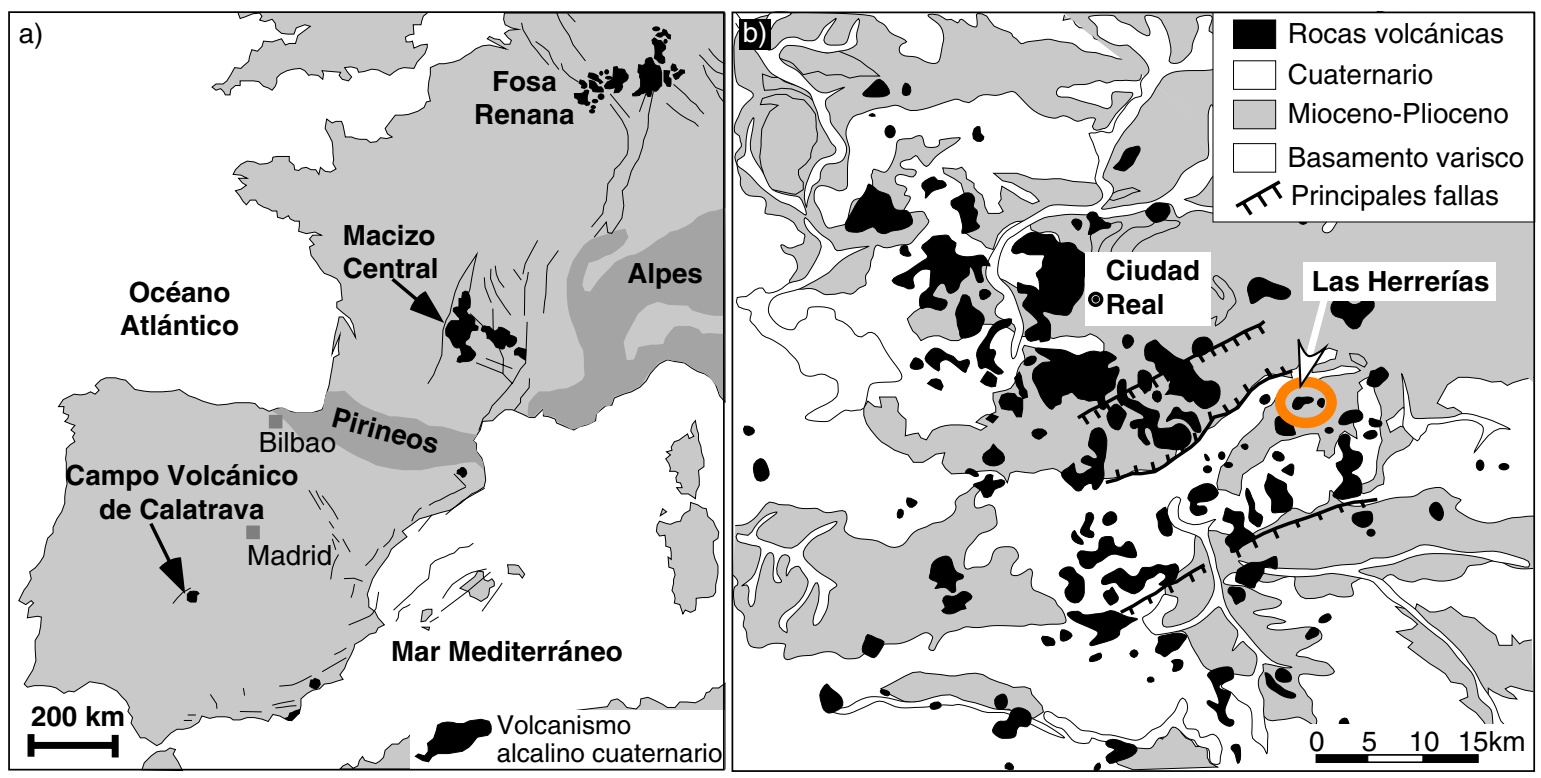

Fig. 1.-A) Principales regiones volcánicas del Terciario-Cuaternario en Europa occidental (modificado de Martí et al., 2011). B) Localización del volcán Las Herrerías en el marco de la Región Volcánica del Campo de Calatrava (modificado de Cebriá \& López Ruiz, 1995). 
al suelo más fríos, pero a temperaturas lo suficientemente elevadas como para aglutinarse (soldarse), dando lugar a depósitos de spatter (conos o rampas de spatter) si la tasa de acumulación es baja o coladas de lava desenraizadas si la tasa de acumulación es alta (Head \& Wilson 1989). Una pequeña cantidad del material emitido es de tamaño ceniza - lapilli medio y es arrastrado convectivamente por encima de la fuente de lava, depositándose más tarde como tefra de caída a una cierta distancia del foco volcánico $(<3 \mathrm{~km})$. Entre los componentes de esta tefra de caída se encuentran clastos fluidales generados por el enfriamiento y solidificación de spray de lava (achnelitos), como por ejemplo las lágrimas y los cabellos de Pele (e.g. Ladle, 1978; Porrit et al., 2012).

El volcán Las Herrerías es un cono de spatter y escorias edificado sobre un maar (Fig. 2). En este volcán afloran depósitos de spatter, lavas y tefras achnelíticas (Carracedo Sánchez et al., 2014). Como anteriormente se ha indicado, tanto el spatter como las tefras achnelíticas son productos típicos de las fuentes de lava hawaianas. Las lavas podrían ser simplemente efusivas, pero en este caso presentan signos obvios de haber sido generadas por coalescencia de piroclastos muy fluidos en torno a una fuente de lava.

En este trabajo se presentan y discuten los primeros datos químicos de las coladas y los depósitos piroclásticos alimentados por fuentes de lava del volcán Las Herrerías, con el objetivo de: (1) incrementar y actualizar el banco de datos químicos de las rocas volcánicas de la RVCC, y (2) comparar la composición química de estos depósitos con la del resto de las lavas de la RVCC, tradicionalmente consideradas como efusivas, es decir emitidas directamente

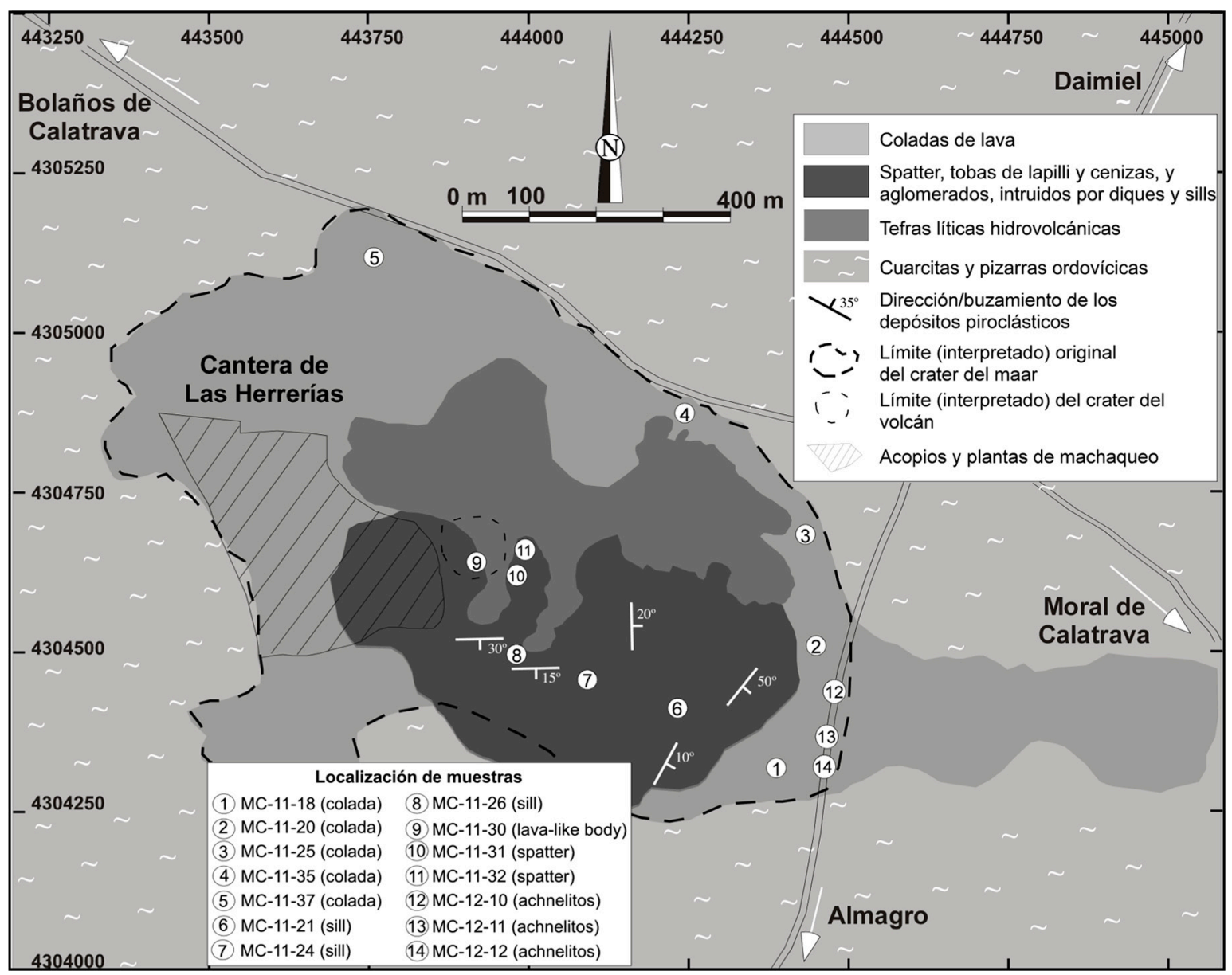

Fig. 2.-Mapa geológico del volcán Las Herrerías en el que también se han añadido la localización de las muestras analizadas en este trabajo. 
desde un foco. El presente trabajo se enmarca dentro de un proyecto de investigación sobre los depósitos hawaianos en la RVCC, uno de cuyos objetivos es tratar de establecer criterios (de campo, petrográficos, químicos, etc.) que permitan identificar coladas clastogénicas (lava-like flows) generadas a partir de fuentes de lava.

\section{El volcán de Las Herrerías}

En 1985, Ramírez Merino et al. cartografiaron en el paraje de Las Herrerías un centro de emisión del que partían dos coladas, una en dirección Norte de escasos 600 metros (melilitas olivínicas; Ramírez Merino et al. 1985), y otra en dirección Este de $1 \mathrm{~km}$ de recorrido (nefelinitas olivínicas; Ramírez Merino et al. 1985). La posterior puesta en marcha de una explotación minera que beneficia las lavas cartografiadas ha permitido detectar nuevos depósitos y reconstruir la compleja actividad de este volcán (Fig. 3A).

La primera fase eruptiva fue freatomagmática, generando un maar y depósitos formados por tobas de cenizas líticas, con una estratificación/laminación planar muy continua. Intercalados en estos depósitos hidrovolcánicos se encuentran varios niveles $(3-10 \mathrm{~cm})$ de tefras achnelíticas compuestas por clastos fluidales, escoriáceos y mixtos (Carracedo Sánchez et al. 2014). Estos depósitos piroclásticos (Fig. 3B), entre los que aparecen lágrimas y cabellos de Pele, son un producto típicamente hawaiano (e.g. Ladle, 1978; Porrit et al., 2012) generado en pulsos de tipo fuente de lava que preceden a la instalación de una fuente más intensa dentro del propio maar, lo que culmina con la construcción de un pequeño cono de spatter.

La pila de spatter pasa gradualmente de techo a muro desde una facies oquerosa, en la que la soldadura se desarrolla esencialmente por aglutinación (Fig. 4A), hasta una facies muy densa (Fig. 4B), en la cual la soldadura se realiza esencialmente por coalescencia (sin contornos inequívocos de clastos) pero que tiene abundantes indicios que permiten conocer o inferir la existencia de clastos originales (vesiculación en parches, fantasmas escoriáceos, contornos de clastos deducibles por partición o alteración selectiva, soldadura incompleta de clastos, abundantes fragmentos de fenocristales, etc.). Por debajo, debido a la coalescencia completa de los piroclastos fluidos, el spatter se transforma gradualmente, aunque de forma rápida (tramo con espesor de 15-40 cm), en una masa de lava coherente, sin signos de clastos (lava-like body en el sentido de Valentine et al., 2000). No obstante, dentro de esta masa de lava coherente hay localmente indicios de clastos que ponen de manifiesto su origen clástico. Todos estos cambios en las características del spatter así como su transición a la masa de lava coherente son muy similares a los descritos en los depósitos generados por fuentes de lava (e.g. Sumner, 1998; Valentine et al., 2000; 2006; Carracedo et al., 2012).

Las erupciones hawaianas dejaron paso finalmente a una fase estromboliana, representada por tefras de lapilli escoriáceo, bombas y fragmentos de bombas (Fig. 3C, D). Por último, la actividad eruptiva culminó con la efusión de lavas desde la base del cono volcánico (Fig. 4C). Estas coladas de lava están conectadas lateralmente con la masa de lava coherente formada por coalescencia de piroclastos fluidos y ubicada en la parte interna del edificio volcánico, por medio de una intrincada red de diques y sills que intruyen en los depósitos piroclásticos hawaianos y estrombolianos que forman el propio cono volcánico (Fig. 4D).

En resumen, las relaciones de campo sugieren que la masa de lava generada por coalescencia de piroclastos fluidos en la base de la fuente de lava intramaar es confinada inicialmente en el interior del cono de spatter. Más tarde, y debido a la presión ejercida sobre la lava por la pila piroclástica (spatter+depósitos estrombolianos), se rompe el cono de spatter y escorias, y la lava sale al exterior a través de los diques y sills, formando así las coladas de lava.

\section{Petrografía de los depósitos alimentados por fuentes de lava}

Las rocas que conforman la masa de lava coherente, las coladas de lava y el conjunto de diques $\mathrm{y}$ sills son de aspecto aparentemente coherente y homogéneo, con estructura masiva y fábrica isótropa. Estas rocas son ultramáficas y presentan, en general, una textura holocristalina, porfídica, con una matriz microcristalina pobremente vesicular (cf., Houghton \& Wilson, 1989). Los fenocristales $(<2 \mathrm{~mm})$ son de olivino $y$, en menor cantidad, piroxeno (augita, diópsido) y melilita, siendo habitual la existencia de 

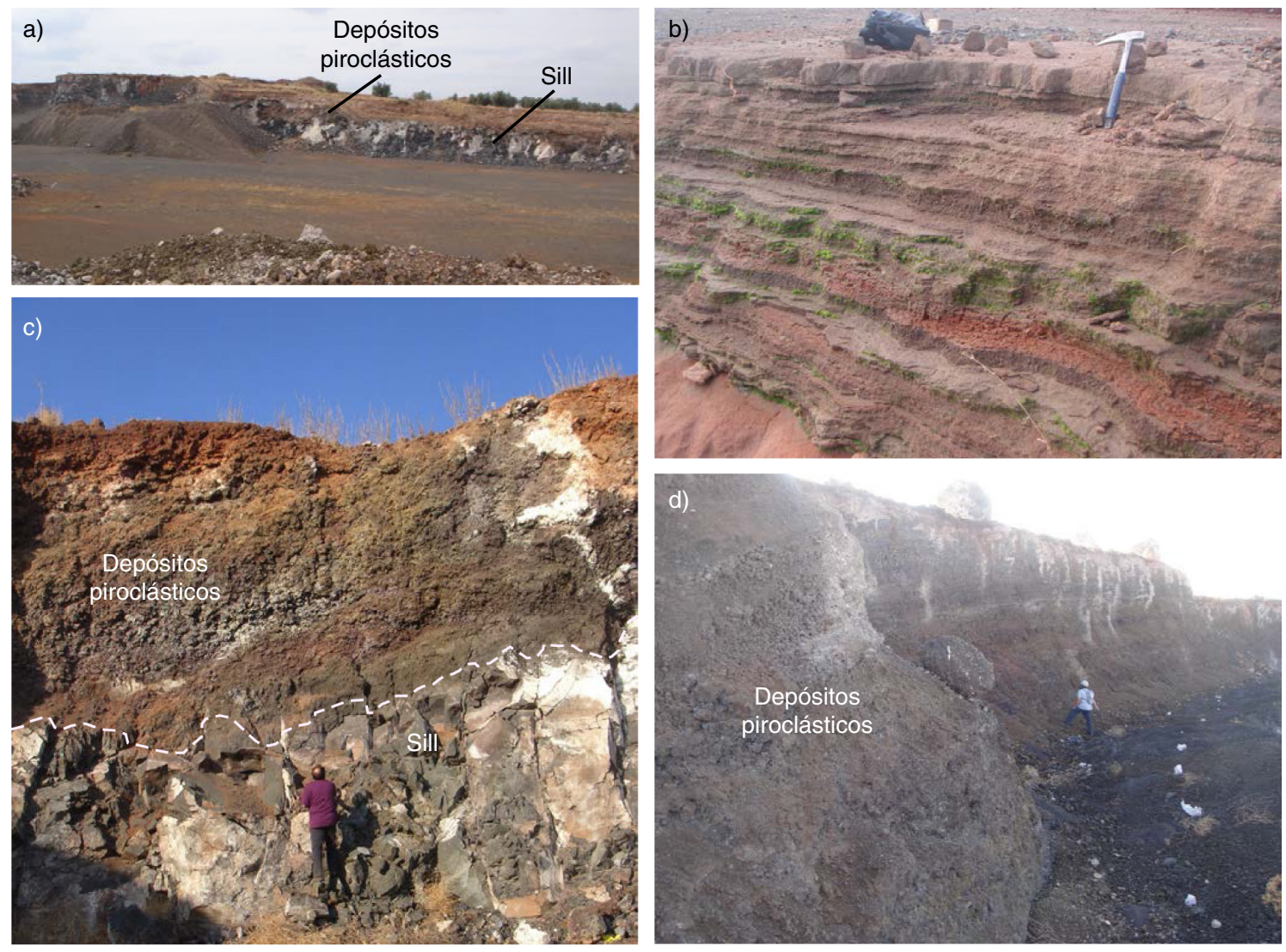

Fig. 3.-A) Aspecto general de la corta Sur de la explotación Las Herrerías. Las superficies blancas en el frente de extracción son pátinas de carbonato cálcico sobre las rocas que conforman la intrusión del sill. B) Aspecto general de las tobas hidrovolcánicas en la base del cono volcánico. C) Intrusión en las tefras de escoria de un sill alimentado por la masa de lava clastogénica. D) Detalle de la potencia de la tefra de escorias y fragmentos de bombas en la parte central del cono volcánico.

fragmentos de estos fenocristales. La matriz de estas rocas está formada por microlitos de clinopiroxeno, magnetita, ilmenita, olivino y nefelina intersticial, con cantidades accesorias de calcita primaria y melilita (Fig. 5A, B). Esta matriz es ligeramente más granuda en las coladas de lava. Las vesículas muchas veces aparecen rellenas por zeolitas y calcita.

No obstante, una observación detallada de estas rocas a nivel petrográfico también pone de manifiesto su origen fragmental, encontrándose entre otros los siguientes indicios: 1) presencia de fragmentos de fenocristales, 2) variaciones locales en la textura y granulometría de la matriz (granular a fluidal), 3) variaciones en la vesiculariad (10-40\%), y 4) variaciones en la distribución de los fenocristales. Por otro lado, las rocas que conforman el depósito de spatter presentan la misma mineralogía que las rocas coherentes, incluso las características de los fenocristales son iguales, sin embargo la matriz tiene una textura más variable, con términos que van, de mayor a menor abundancia, desde holohialinos (taquilítica), pasando por criptocristalinos hasta microcristalinos (Fig. 5C). Además, la vesicularidad de estas rocas también es mucho más variable $(10 \%-80 \%)$.

Finalmente, los achnelitos que conforman las tefras achnelíticas intercaladas entre los depósitos hidrovolcánicos, se caracterizan por mostrar una textura hialocristalina porfídica, con matriz microlítica, que en algunos casos es fluidal, variablemente vesicular $(0 \%-50 \%)$. Los fenocristales son idio-hipidiomorfos y se corresponden esencialmente con cristales de olivino, aunque también hay algunos de clinopiroxeno, mientras que los microlitos de la matriz son esencialmente de clinopiroxeno (Fig. 5D). El vidrio de la matriz es mayoritariamente sideromelánico, aunque ocasionalmente en zonas puntuales se ha encontrado vidrio taquilítico. 


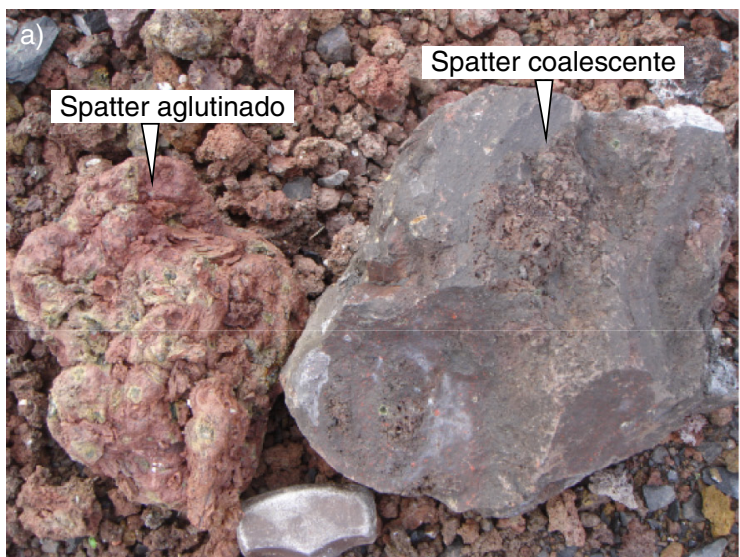

c)

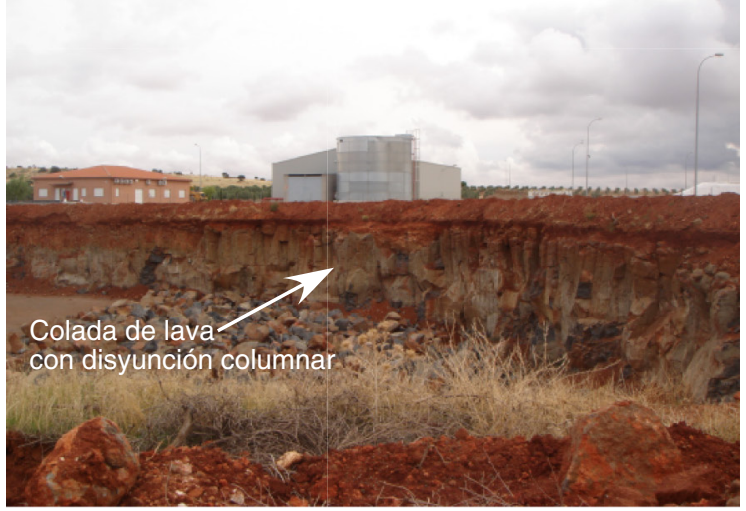

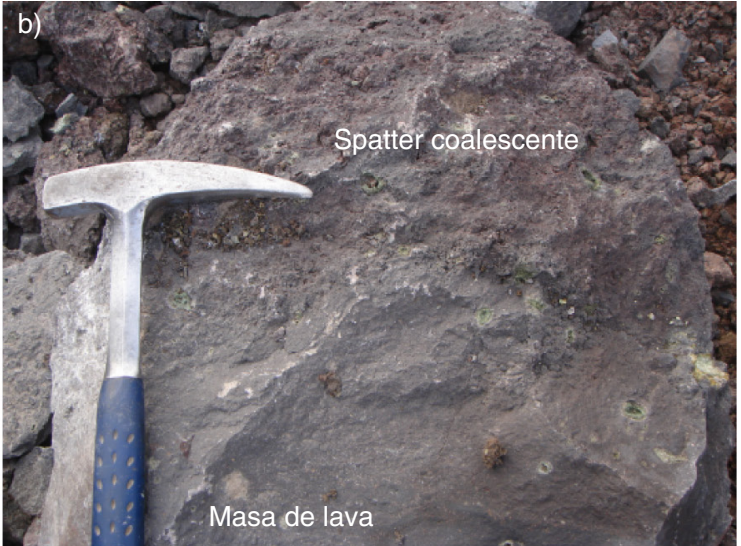

d)
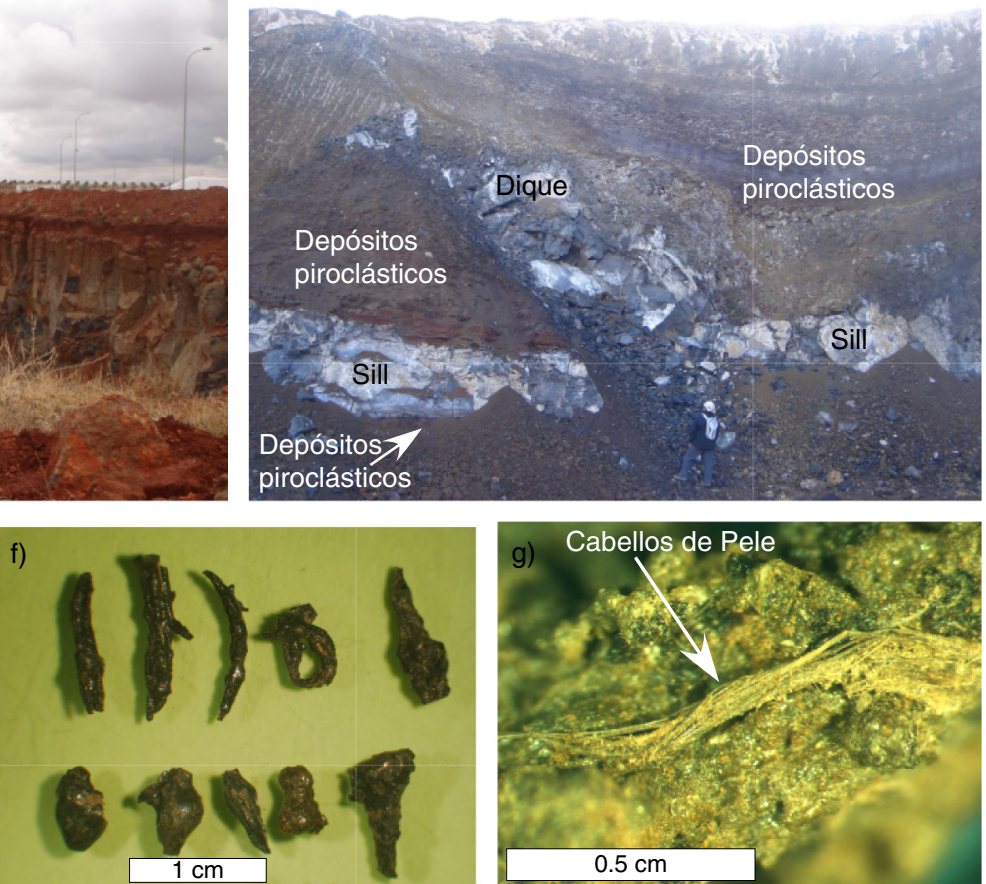

Fig. 4.-Depósitos generados a partir de una fuente de lava del volcán Las Herrerías. A) Contraste estructural entre el spatter aglutinado (rico en huecos entre clastos y con claros límites de clastos) y el spatter coalescente (prácticamente carente de límites de clastos y escaso en huecos). B) Detalle de la transición rápida desde el spatter coalescente a la masa de lava clastogénica (lava-like mass). C) Colada de lava clastogénica, fuera ya del cono volcánico, con una marcada disyunción columnar. D) Dique sin-volcánico enraizado en el sill e intrusivo en los depósitos piroclásticos estrombolianos generados tras los episodios hawaianos. E y F) Detalle de los achnelitos esferoidales y elongados. G) Detalle de los cabellos de Pele.

\section{Métodos analíticos}

Para llevar a cabo la caracterización geoquímica de las rocas formadas en la fuente de lava se han seleccionado 13 muestras, de las que 9 corresponden a lavas: coladas de lava (5), sills/diques (3) y masa de lava (1). De las cinco restantes, 2 son de spatter y
3 de tefras achnelíticas intercaladas en los depósitos hidrovolcánicos.

Los análisis químicos se han realizado en el Servicio de Geocronología y Geoquímica (SGIker) de la Universidad del País Vasco UPV/EHU por espectrometría de masas (ICP-masas; Thermo Scientific XSeries II), siguiendo el método de García 

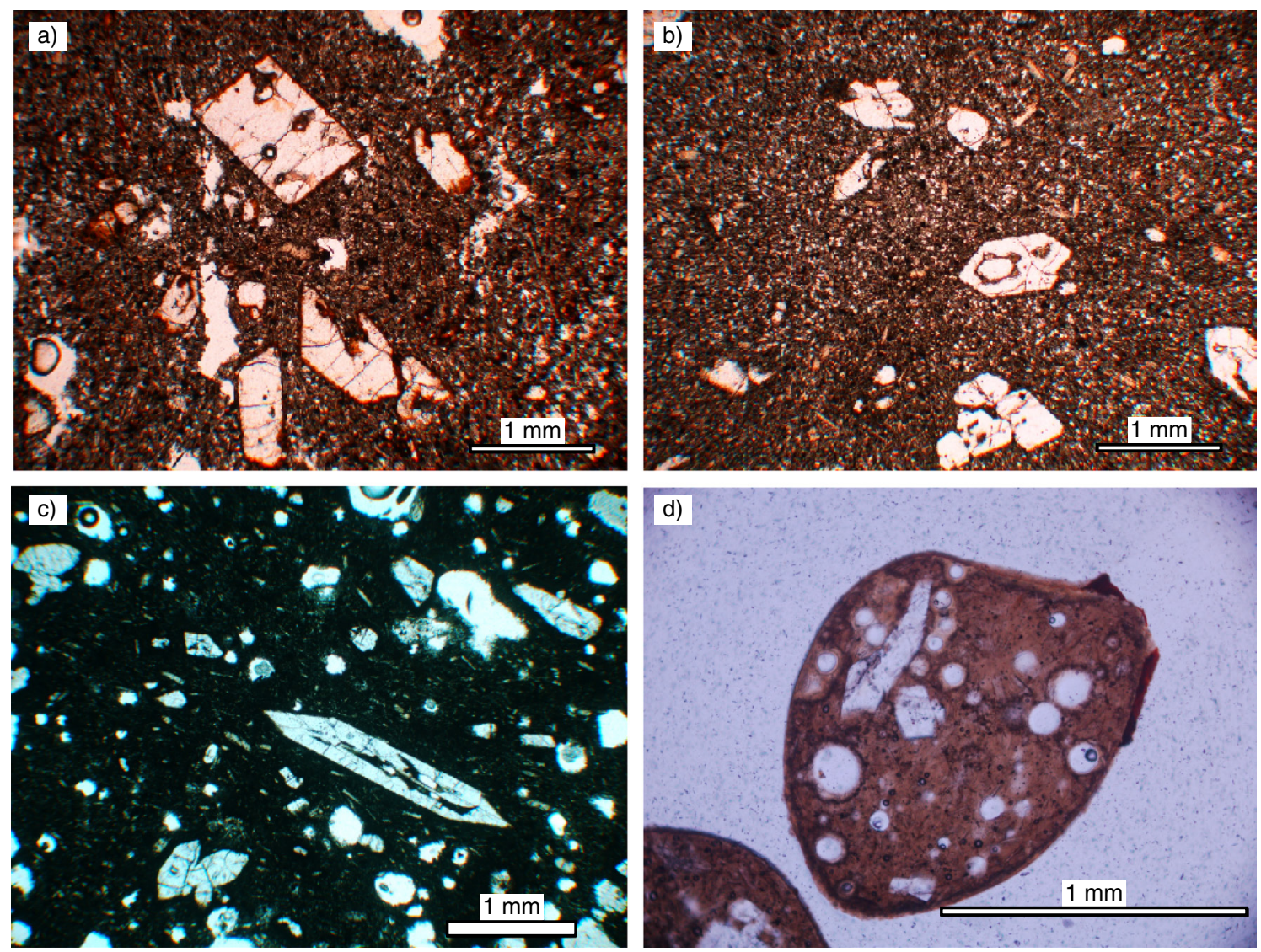

Fig. 5.-Microtexturas de las rocas alimentadas por fuentes de lava del volcán Las Herrerías obtenidas de los distintos tipos de depósitos estudiados. A) Sill. B) Colada de lava. C) Spatter. D) Achnelito esferoidal ("lágrima de Pele”).

de Madinabeitia et al. (2008). Los resultados analíticos se muestran en las Tablas 1 y 2.

\section{Geoquímica de elementos mayores y trazas}

Los datos químicos obtenidos (Tablas 1 y 2) indican que todas las muestras son composicionalmente similares, independientemente del tipo de depósito considerado. Solamente las tefras achnelíticas se separan del rango composicional definido por el resto de depósitos hawaianos, aunque probablemente esto sea debido a su avanzado estado de alteración (LOI=4.13-10.72\%); es por ello que, a pesar de que en este trabajo se han proporcionado sus composiciones químicas (Tablas 1 y 2), estas tefras han sido excluidas de la discusión geoquímica. Se trata de rocas ultrabásicas $\left(\mathrm{SiO}_{2}=35.55-39.52 \%\right)$ fuertemente alcalinas $\left(\mathrm{Na}_{2} \mathrm{O}+\mathrm{K}_{2} \mathrm{O}=3.71-6.09 \%\right)$ y sódicas $\left(\mathrm{Na}_{2} \mathrm{O}=2.24-3.81 \%\right)$. Presentan bajos contenidos en aluminio $\left(\mathrm{Al}_{2} \mathrm{O}_{3}=9.64-10.99 \%\right)$ y altos contenidos en magnesio $(\mathrm{MgO}=11.58-14.19 \%)$, hierro $\left(\mathrm{Fe}_{2} \mathrm{O}_{3}{ }^{(\mathrm{t})}=11.88-15.22 \%\right)$, titanio $\left(\mathrm{TiO}_{2}=2.52-2.85 \%\right)$ y calcio $(\mathrm{CaO}=12.31-15.18 \%)$. Los valores de pérdida por calcinación (LOI) son relativamente bajos (1.42-3.88\%), teniendo en cuenta que son rocas altas en magnesio.

De acuerdo con la clasificación TAS (Fig. 6A) todas las muestras se clasifican como foiditas, aunque considerando que los contenidos normativos de nefelina y albita son inferiores al $20 \%$ y $5 \%$, respectivamente, estas rocas deben clasificarse como melanefelinitas (Le Maitre et al., 2002).

A diferencia de los elementos mayores, las concentraciones de la mayor parte de los elementos traza son variables de unas muestras a otras (Tabla 2). En líneas generales se puede afirmar que los contenidos en algunos elementos traza, tales como Cr (325520 ppm), Ni (169-286 ppm), V (126-293 ppm), 


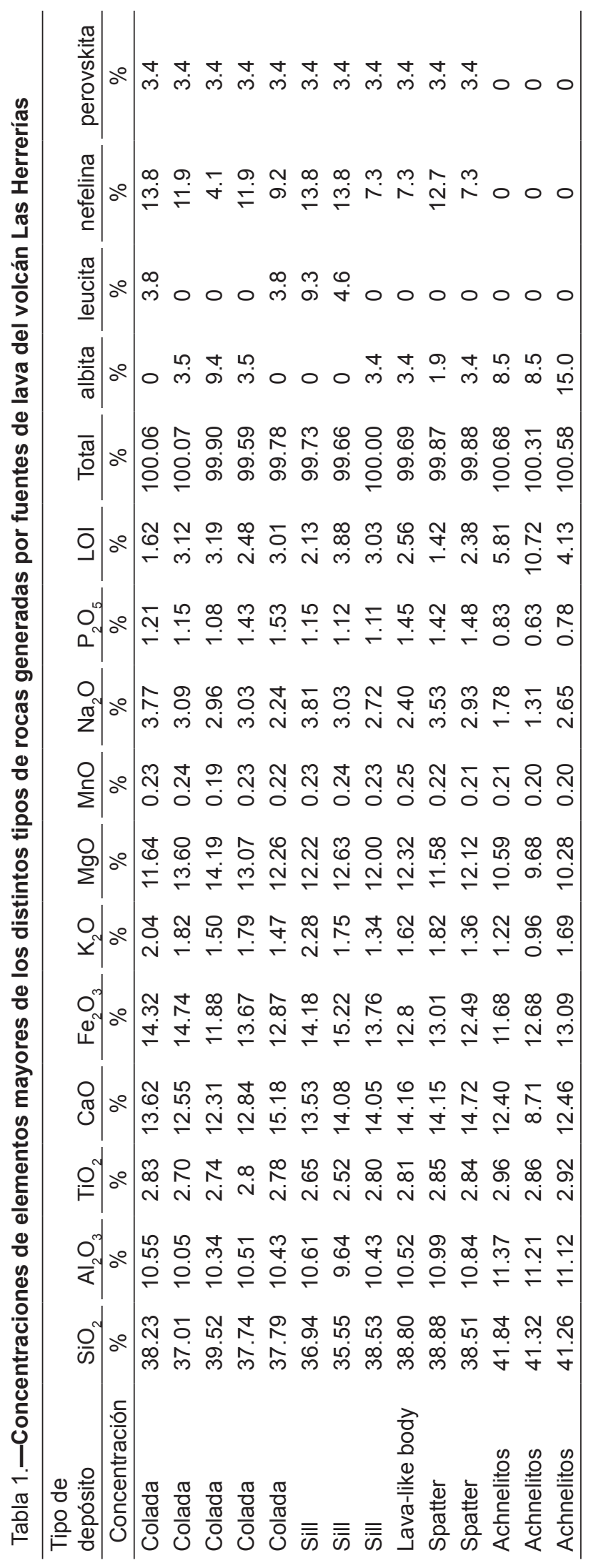

Zr (283-374 ppm), Y (25-37 ppm) y Th (9-16 ppm) son relativamente moderados a bajos a tenor del carácter ultrabásico de estas rocas y de sus concentraciones relativamente altas en $\mathrm{Nb}(94-110 \mathrm{ppm})$ y Ta (6-11 ppm). Por otro lado, se observan marcados enriquecimientos en elementos de gran radio iónico (LILE), especialmente Ba (604-1200 ppm), $\mathrm{Rb}(21-46 \mathrm{ppm})$ y $\mathrm{Sr}(783-1746 \mathrm{ppm})$, frente a los elementos con alto potencial iónico (HFSE). No obstante, dentro de estos últimos las concentraciones totales de tierras raras ( $\mathrm{REE}$ ) son relativamente altas (371-431ppm), mostrando importantes enriquecimientos $(10 \mathrm{x}-200 \mathrm{x})$ con respecto al condrito (Sun \& McDonough, 1989). Los perfiles normalizados (Fig. 6B) muestran un mayor enriquecimiento en las tierras raras ligeras (LREE) que en las pesadas (HREE), con acusados grados de fraccionación de $\operatorname{REE}\left[\left((\mathrm{La} / \mathrm{Lu})_{\mathrm{N}}=32-35\right)\right]$. No se observan anomalías negativas en $\mathrm{Eu}$ (Fig. 6B).

Los diagramas multielementales normalizados frente al manto primitivo de Sun \& McDonough (1989) muestra un progresivo enriquecimiento de los elementos más incompatibles (desde el Rb hasta el Ta) y un decrecimiento en los más compatibles, con marcadas anomalías negativas en $\mathrm{K}$ y, en menor medida, Sr y Zr (Fig. 6C). Estos espectros, junto con las relaciones elementales $\mathrm{La} / \mathrm{Nb}(0.8-1.0)$, $\mathrm{Zr} / \mathrm{Nb}$ (2.7-3.5), $\mathrm{Rb} / \mathrm{Nb}(0.2-0.4), \mathrm{Th} / \mathrm{Nb}(0.1)$, $\mathrm{Nb} / \mathrm{Y}(2.5-3.4)$ y Zr/Y (8.5-9.1) son típicos de basaltos de isla oceánica (OIB) poniendo de manifiesto la procedencia de estas rocas a partir de un manto enriquecido (Sun \& McDonough, 1989; Weaver, 1991; Condie, 2005).

Teniendo en cuenta la escasa variabilidad en sílice del conjunto de muestras estudiadas, y considerando que las potenciales fases mayoritarias que pudieran fraccionarse son olivino, piroxeno y plagioclasa, se ha seleccionado el Zr para la construcción de algunos diagramas de variación (Fig. 7), ya que es un elemento incompatible para dichas fases. En estos diagramas se observa que el progresivo enriquecimiento en $\mathrm{Zr}$ lleva consigo enriquecimientos en $\mathrm{P}_{2} \mathrm{O}_{5}, \mathrm{CaO}, \mathrm{Al}_{2} \mathrm{O}_{3}, \mathrm{TiO}_{2}$ e Y, entre otros elementos, $\mathrm{y}$ ligeros empobrecimientos en $\mathrm{Fe}_{2} \mathrm{O}_{3}$ y MgO, mientras que las concentraciones en $\mathrm{Ni}, \mathrm{Cr}, \mathrm{Nb}, \mathrm{SiO}_{2}$ y álcalis se mantienen constantes.

Finalmente, las composiciones obtenidas son típicas del volcanismo desarrollado en zonas de 

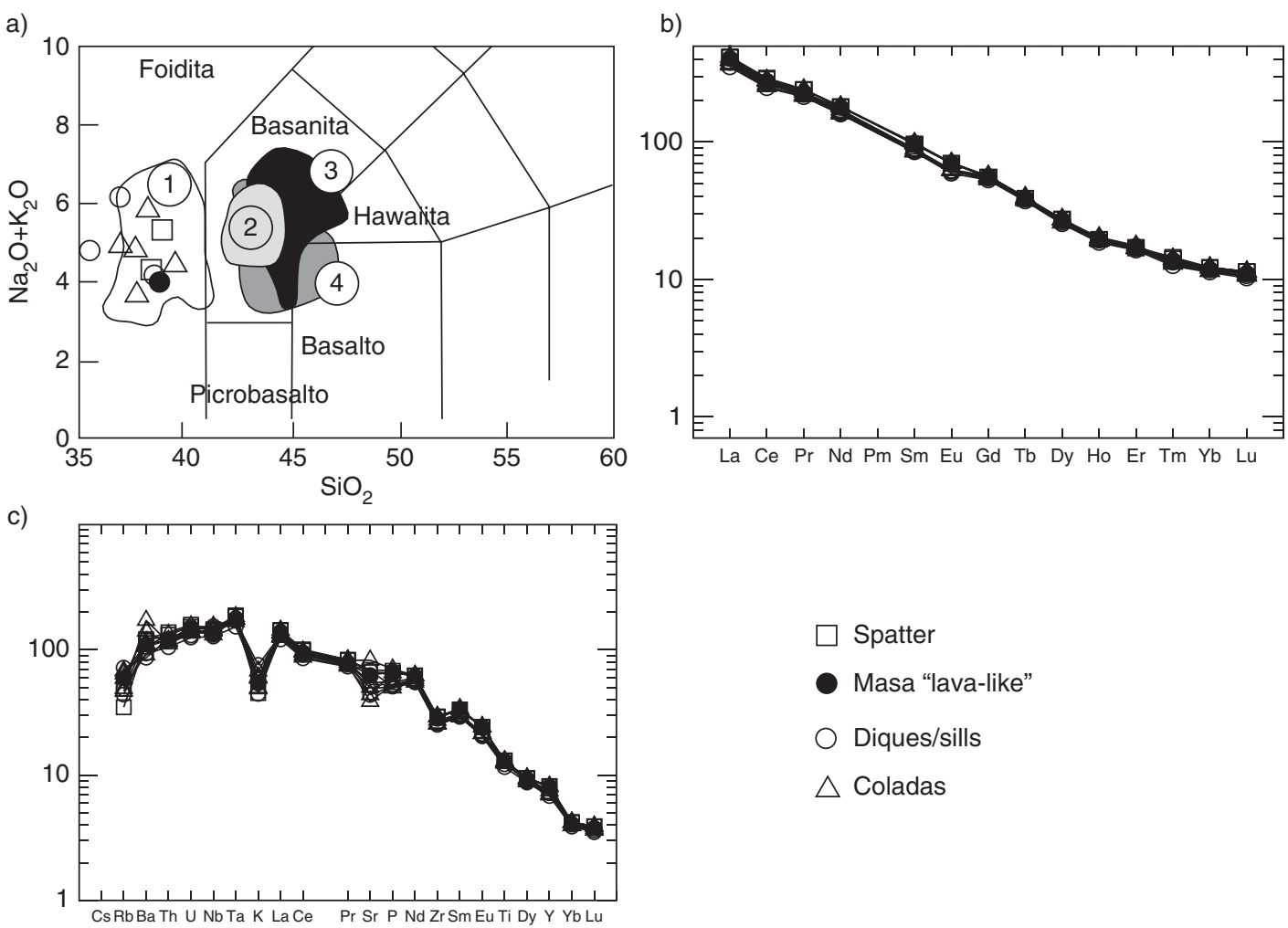

Fig. 6.-A) Proyección de las muestras estudiadas en el diagrama TAS (Le Maitre et al., 2002). Los campos numerados se corresponden con las rocas de la RVCC estudiadas por Cebriá \& López Ruiz (1995): 1) Melilititas, 2) Nefelinitas, 3) Basaltos olivínicos y 4) Leucititas. B y C) Diagramas de normalización de REE y multielementales frente al condrito y el manto primitivo de Sun \& McDonough (1989), respectivamente.

intraplaca continental, tal y como se desprende de las proyecciones en distintos diagramas de discriminación tectonomagmática, como por ejemplo el Ti/100-Zr-3Y (Pearce \& Cann, 1973), el $\mathrm{Th}-\mathrm{Zr} / 117-\mathrm{Nb} / 16$ (Wood, 1980) o el 2Nb-Zr/4-Y (Meschede, 1986); en todos estos diagramas las muestras analizadas se proyectan en los campos de los basaltos de intraplaca y de los basaltos alcalinos de intraplaca.

\section{Discusión}

Los datos químicos obtenidos para los depósitos alimentados por fuentes de lava del volcán Las Herrerías entran dentro del rango composicional de las lavas alcalinas de la RVCC (e.g. Ancochea, 1982; Cebriá, 1992; Cebriá \& López Ruiz, 1995; López Ruiz et al., 2002; Ancochea, 2004). Concretamente, en el diagrama TAS se proyectan mayoritariamente en el campo de las melilititas definido por
Cebriá \& López Ruiz (1995). Sus bajos contenidos en $\mathrm{SiO}_{2}$ y $\mathrm{Al}_{2} \mathrm{O}_{3}$ (Tabla 1) las equiparan efectivamente a las melilititas caracterizadas por Cebriá (1992): $\mathrm{SiO}_{2}=36.17-40.71 \%$ y Al${ }_{2} \mathrm{O}_{3}=8.97-13.15 \%$. Aplicando los criterios de la IUGS (Le Maitre et al., 2002), tanto las rocas de Las Herrerías como aquellas empleadas para su comparación (Cebriá, 1992) se clasifican como melanefelinitas. Dos de las muestras presentan melilita modal accesoria, aunque carecen de larnita normativa, por lo que no se pueden clasificar como melilititas. La clasificación química obtenida es congruente con la existencia de nefelina en todas las muestras.

Se ha recalculado la mineralogía normativa de las melilititas caracterizadas por Cebriá (1992) para su comparación con la de las muestras de Las Herrerías. Es en este aspecto donde surgen las mayores discrepancias, puesto que de las 38 muestras de melilititas presentadas por Cebriá (1992), aunque todas tengan menos del $20 \%$ en nefelina 


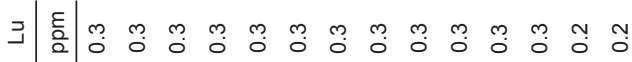

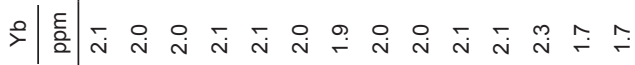

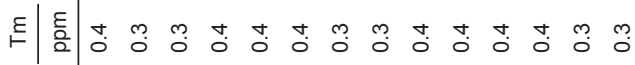

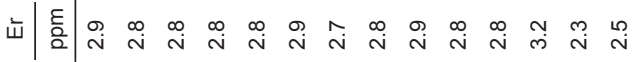

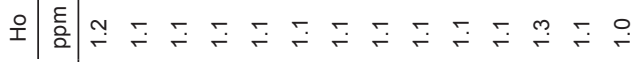

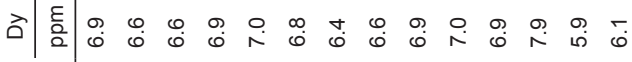

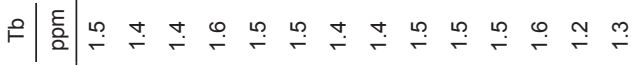
ర)

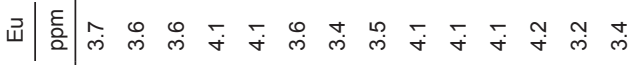

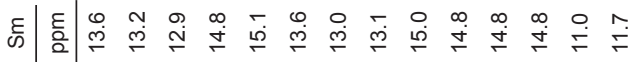

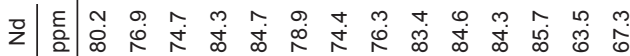

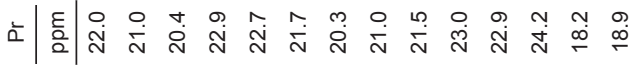
४

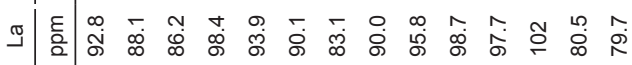

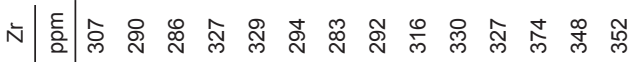

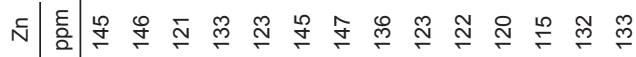

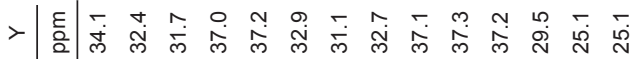
$>\mid \frac{0}{2}$ 용 \& 원

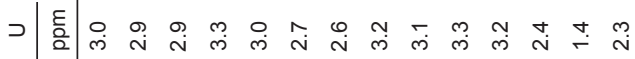

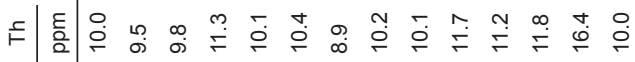

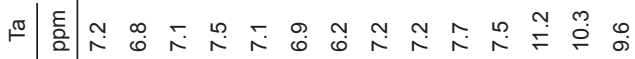
i) ஸ) व)

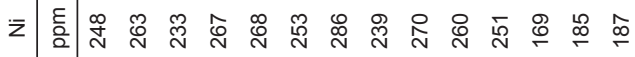

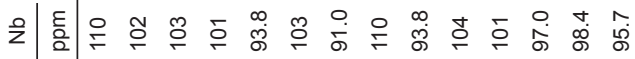

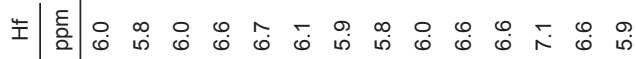

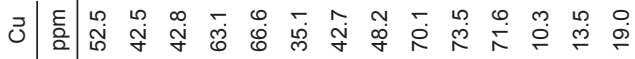
广

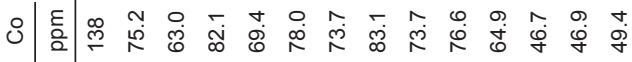
๓ .

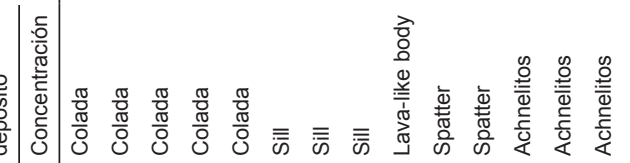

normativa, solamente dos tienen más de un $10 \%$ en larnita, el resto presentan este mineral en cantidades variables $(0 \%-6.8 \%)$ pero siempre por debajo del $10 \%$, con lo que principalmente se clasificarían como nefelinitas melilíticas. Por otra parte, los contenidos en leucita son mayores $(\mathrm{Lc}=3.7 \%)$ en las muestras estudiadas por Cebriá (1992) que en las de Las Herrerías ( $\mathrm{Lc}=2.0 \%$ ); además, en estas últimas siempre aparece perovskita normativa (3.4\%), mineral ausente en las muestras estudiadas por Cebriá (1992).

Los diagramas de normalización de REE (Fig. 6B) y los multielementales (Fig. 6C) frente al condrito y al manto primitivo de Sun \& McDonough (1989) proporcionan unos espectros normalizados casi idénticos a los de las melilititas estudiadas por Cebriá (1992), si bien las muestras de Las Herrerías tienen espectros de REE ligeramente más fraccionados ((La/ $\mathrm{Lu})_{\mathrm{N}}=33-35$ ) y mayores enriquecimientos en $\mathrm{K}$ (Fig. 6C). Por otro lado, todas las rocas de Las Herrerías analizadas presentan contenidos en sílice $<50 \%$, tienen números de magnesio moderados a relativamente bajos $\left(\mathrm{Mg}^{*}=45-54\right)$, acordes de alguna forma con los bajos contenidos en $\mathrm{Ni}(233-286 \mathrm{ppm})$ y $\mathrm{Cr}$ (393-520 ppm), con lo cual no se corresponden con composiciones de magmas primarios (Wilson, 1989). Sin embargo, tal como indica Cebriá (1992) los contenidos de $\mathrm{Cr}$ y Ni en los magmas primarios pueden variar en función de la mineralogía del manto y de los coeficientes de distribución, por lo que la elección de dichos valores se debe basar en el rango de variación presente en las rocas objeto de estudio. Por otro lado, para el cálculo del número de magnesio en las muestras de Las Herrerías no se ha podido considerar el FeO, mientras que Cebriá (1992) para el cálculo de $\mathrm{Mg}$ * iguala la relación $\mathrm{Fe}_{2} \mathrm{O}_{3} / \mathrm{FeO}$ a 0.15 , obteniendo principalmente valores de $\mathrm{Mg}^{*}>68$ en las melilititas, considerándolas como composiciones primarias. No obstante, las relaciones $100 * \mathrm{MgO} /\left(\mathrm{MgO}+\mathrm{Fe}_{2} \mathrm{O}_{3}\right)$ de las melilititas estudiadas por Cebriá (1992), comprendidas en el rango 56-80, son mayores que las obtenidas en Las Herrerías, por lo que posiblemente estas últimas hayan sufrido una previa fraccionación (olivino?). Esta circunstancia también podría explicar la ausencia de enclaves del manto en las rocas de Las Herrerías, criterio empleado en determinadas ocasiones para la caracterización de magmas primarios Maaløe (1973). 

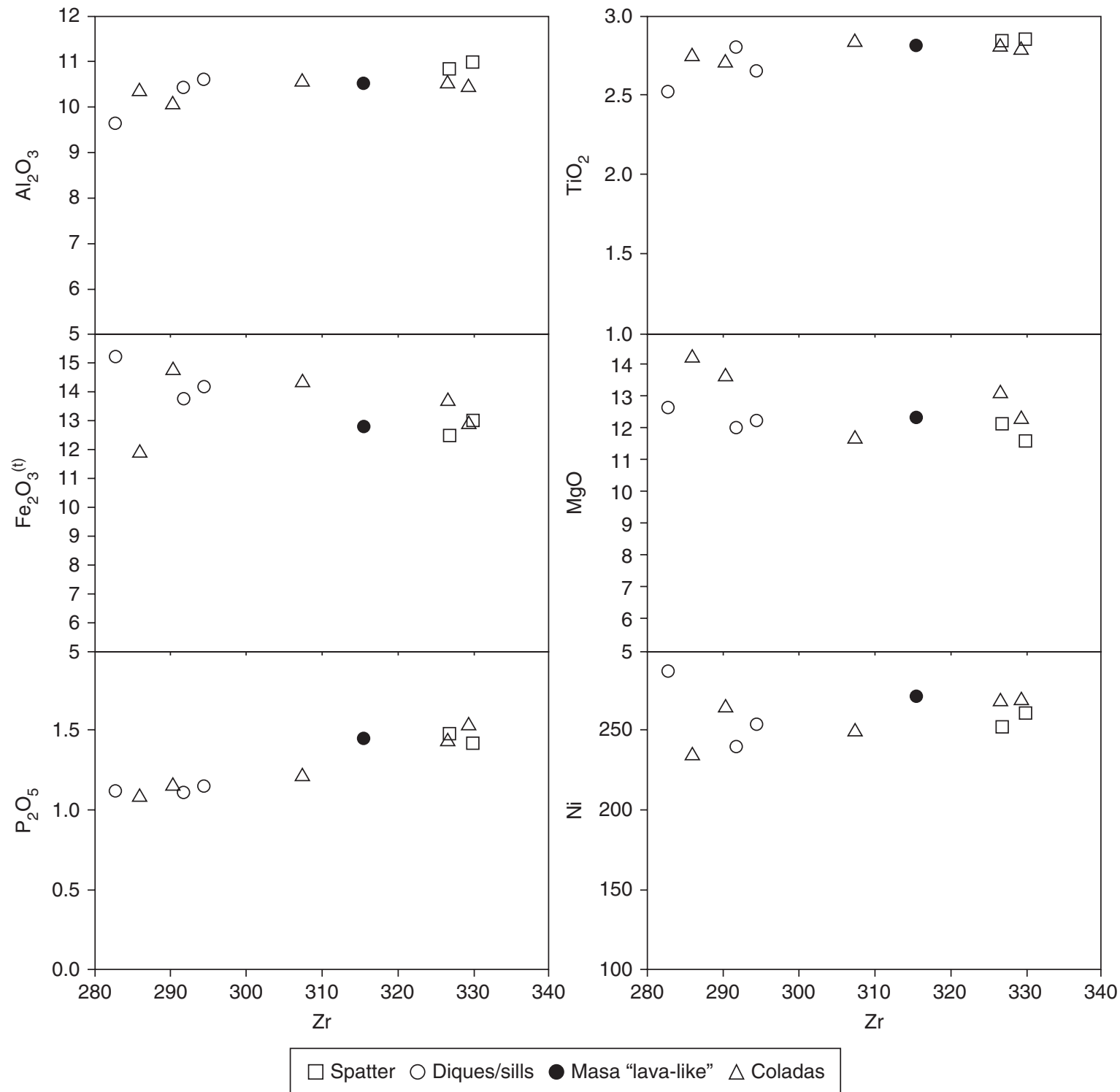

Fig. 7.-Diagramas de variación frente al $\mathrm{Zr}$ de algunos elementos mayores y traza seleccionados, correspondientes a los distintos depósitos alimentados por fuentes de lava del volcán Las Herrerías.

No obstante, a pesar de que el conjunto estudiado no presenta composiciones primarias, sí exhibe ligeras variaciones composicionales (Tablas 1 y 2 ). Tal y como se ha adelantado anteriormente, los trends de ligero empobrecimiento con respecto al $\mathrm{Zr}$ en $\mathrm{Fe}_{2} \mathrm{O}_{3}$ y $\mathrm{MgO}$, y los enriquecimientos o los trends horizontales con respecto al $\mathrm{Zr}$ del resto de los elementos analizados, serían compatibles con la fraccionación de clinopiroxeno (Fig. 7). Si las variaciones composicionales derivaran del mecanismo de fusión, este hecho quedaría reflejado, por ejemplo, en el comportamiento de la evolución del $\mathrm{Zr}$ e Y. Ambos elementos son incompatibles para las potenciales fases fraccionables, por lo que la relación $\mathrm{Zr} / \mathrm{Y}$ nos informaría de las características del proceso de fusión. En este sentido, como el Zr es más incompatible en las fases del manto que el Y (Nicholson \& Latin, 1992) la relación $\mathrm{Zr} / \mathrm{Y}$ tiende a ser más alta para tasas de fusión bajas. Los valores de Zr/Y (9.5-9.1) para las muestras de Las Herrerías son relativamente altas, lo que indica bajas tasas de fusión, pero además la ausencia de variaciones en 
la relación $\mathrm{Zr} / \mathrm{Y}$ con respecto al contenido en $\mathrm{Zr}$ de las muestras indica que las variaciones químicas en el conjunto de Las Herrerías respondería a un proceso de fraccionación (Fig. 8A). En este sentido, Treuil \& Joron (1975) han demostrado que la relación $\mathrm{La} / \mathrm{Sm}$ frente a $\mathrm{La}$ se mantiene constante durante la cristalización fraccionada, mientras que la fusión en equilibrio proporciona rectas de pendiente negativa. En el caso de las muestras estudiadas se observa una recta horizontal entre dichas relaciones elementales (Fig. 8B), con lo que, una vez más, parece que las diferencias composicionales observadas responderían a los efectos producidos por una ligera fraccionación de clinopiroxeno.

a)

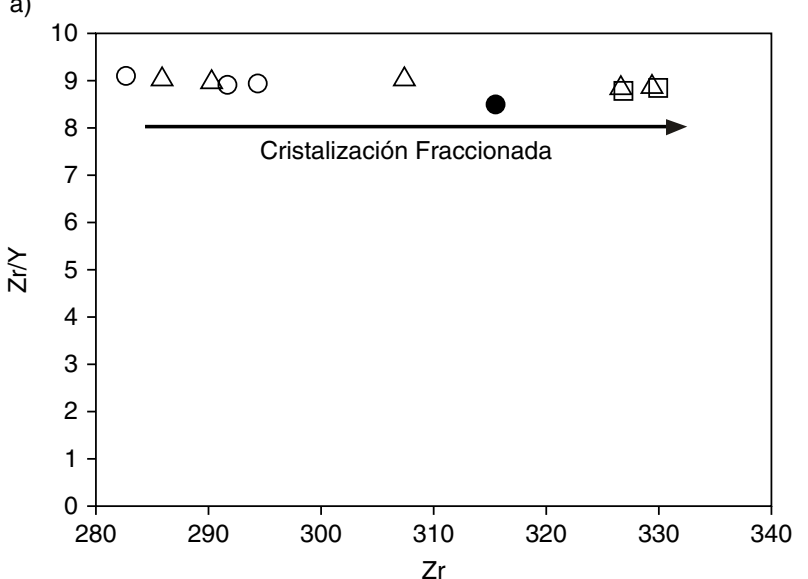

b)

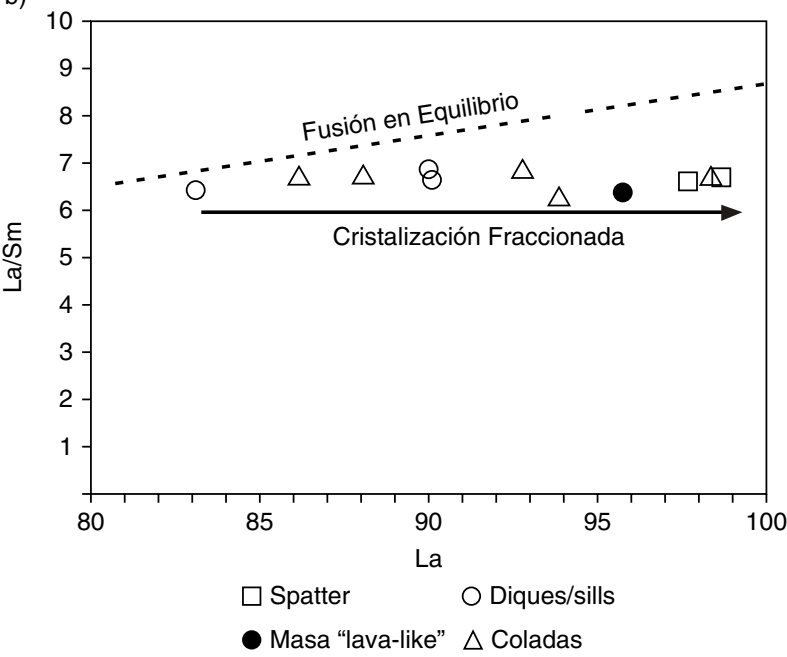

Fig. 8.-A) Diagrama de la relación $\mathrm{Zr} / \mathrm{Y}$ frente a $\mathrm{Zr}$ de los distintos depósitos alimentados por fuentes de lava del volcán Las Herrerías. B) Diagrama La/Sm frente a La para el mismo conjunto de rocas.
Efectivamente, Cebriá (1992) en un estudio más amplio y profundo de la evolución geoquímica del magmatismo de la RVCC, concluyó que los líquidos primarios nefeliníticos procedían de la fusión parcial en condiciones no modales de un manto lherzolítico granatífero con flogopita, y estos líquidos se diferenciaron por la fraccionación de olivino $(2-10 \%)$ y clinopiroxeno (2-18\%). Por tanto, debido a que las melanefelinitas de Las Herrerías no responden a composiciones primarias, las variaciones químicas observadas podrían deberse en gran medida a la fraccionación de clinopiroxeno. No obstante, dentro de cada tipo de depósito también existen variaciones composicionales (Fig. 8A), por lo que concluimos que dichas variaciones reflejan probablemente una distribución heterogénea de este mineral en el magma durante la erupción.

Por tanto, a pesar de las ligeras variaciones composiciones de las melanefelinitas de Las Herrerías, la información petrogenética obtenida por Cebriá (1992) para las melilititas de la RVCC también puede hacerse extensiva a los depósitos estudiados en este trabajo: (1) los líquidos primarios melilitíticos procederían de la fusión parcial en condiciones no modales de un manto lherzolítico granatífero con flogopita (2-9\%, modal), fuertemente enriquecido en elementos traza altamente incompatibles y moderadamente para los elementos con menor grado de incompatibilidad, (2) el porcentaje de fusión estaría situado en torno al 5\%, y (3) la participación del granate en el fundido no habría superado el $2 \%$.

\section{Conclusiones}

Todos los depósitos alimentados por fuentes de lava del volcán Las Herrerías (spatter, diques/sills, masa de lava clastogénica y las coladas de lava) son composicionalmente muy similares, aunque los piroclastos de menor tamaño (achnelitos) no se han podido caracterizar debido a su alteración química (LOI $=4.13-10.72 \%)$. Las rocas que constituyen estos depósitos se corresponden con melanefelinitas ultrabásicas alcalinas fuertemente sódicas, ricas en magnesio, calcio y hierro, y pobres en aluminio. Los concentraciones totales de tierras raras son relativamente altas $(10 \mathrm{x}-200 \mathrm{x}$ condrito), con un mayor enriquecimiento en tierras raras ligeras respecto a las pesadas $\left[(\mathrm{La} / \mathrm{Lu})_{\mathrm{N}}=32-35\right]$. 
Los contenidos en Ni (233-286 ppm), Cr (393$520 \mathrm{ppm})$ y el número de magnesio $\left(\mathrm{Mg}^{*}=45-54\right)$ indican que estos magmas experimentaron una primera fraccionación (olivino \pm ortopiroxeno?) antes de ser emitidos. La variable distribución de clinopiroxeno durante la erupción probablemente condicionó las ligeras variaciones composicionales observadas en las melanefelinitas de Las Herrerías, las cuales fueron emitidas a partir de una fuente de lava en un contexto de intraplaca continental.

\section{AGRADECIMIENTOS}

Trabajo financiado por el Ministerio de Ciencia e Innovación ("Grupo Consolidado", Proyecto CGL2008-01130/BTE) y por la Universidad del País Vasco/EHU (Grupo de Investigación Consolidado GIU12/03). También queremos agradecer las facilidades prestadas por Narciso Cejudo, director de la explotación minera Las Herrerías.

\section{Referencias}

Ancochea, E. (1982). Evolución espacial y temporal del vulcanismo reciente de España Central. Tesis Doctoral, Universidad Complutense de Madrid, 675 pp.

Ancochea, E. (2004). La región volcánica del Campo de Calatrava. In: Geología de España (Vera, J.A., Ed.). SGE-IGME, Madrid, 676-677.

Carracedo Sánchez, M.; Sarrionandia, F.; Arostegi, J. \& Gil Ibarguchi, J.I. (2014). Achnelitos generados en fuentes de lava de la región volcánica de Campo de Calatrava (Cidudad Real, España). Geogaceta, 55: 91-94.

Carracedo Sánchez, M.; Sarrionandia, F.; Arostegui, J.; Eguiluz, L. \& Gil Ibarguchi, J.I. (2012). The transition of spatter to lava-like body in lava fountain deposits: features and examples from the Cabezo Segura volcano (Calatrava, Spain). Journal of Volcanology and Geothermal Research, 227-228: 1-14. http://dx.doi.org/10.1016/j.jvolgeores.2012.02.016.

Cebriá, J.M. (1992). Geoquímica de las rocas basálticas y leucititas de la Región Volcánica de Campo de Calatrava, España. Tesis Doctoral, Universidad Complutense de Madrid, 314 pp.

Cebriá, J.M. \& López Ruiz, J. (1995). Alkali basalts and leucitites in an extensional intracontinental plate setting. Lithos, 35: 27-46. http://dx.doi.org/ 10.1016/0024-4937(94)00027-Y.

Condie, K.C. (2005). High field strength element ratios in Archean basalts: a window to evolving sources of mantle plumes? Lithos 79 : 491-504. http://dx.doi. org/10.1016/j.lithos.2004.09.014.

García de Madinabeitia, S.; Sánchez Lorda, M.E. \& Gil Ibarguchi, J.I. (2008). Simultaneous determination of major to ultratrace elements in geological samples by fusion-dissolution and inductively coupled plasma mass spectrometry techniques. Analytica Chimica Acta, 625: 117-130. http://dx.doi.org/10. 1016/j.aca.2008.07.024.

González, E.; Gosálvez, R.; Becerra, R. \& Escobar, E. (2007). Actividad eruptiva holocena en el Campo de Calatrava (volcán Columba, Ciudad Real, España). Proceedings of XII Reunión Nacional del Cuaternario, AEQUA, Ávila, Spain, 143-144.

Head, JW. \& Wilson, L. (1989). Basaltic pyroclastic eruptions: Influence of gas-release patterns and volume fluxes on fountain structure, and the formation of cinder cones, spatter cones, rootless flows, lava ponds and lava flows. Journal of Volcanology and Geothermal Research, 37: 261-271. http://dx.doi. org/10.1016/0377-0273(89)90083-8.

Houghton, B.F. \& Wilson, C.J.N. (1989). A vesicularity index for pyroclastic deposits. Bulletin of Volcanology, 51: 451-462. http://dx.doi.org/10.1007/ BF01078811.

Ladle, G.H. (1978). Scanning electron microscopy and petrography of glassy particles produced by lava fountains eruptions. Lunar and Planetary Institute, 325: 203ロSS.

Le Maitre, R.W.; Streckeisen, A.; Zanettin, B.; Le Bas, M.J.; Bonin, B.; Bateman, P.; Bellieni, G.; Dudek, A.; Efremova, S.; Keller, J.; Lameyre, J.; Sabine, P.A.; Schmid, R.; Sorensen, H. \& Wolley, A.R. (2002). Igneous Rocks. A Classification and Glossary of terms. Cambridge University Press, 236 pp. http://dx.doi.org/10.1017/CBO9780511535581.

López Ruiz, J.; Cebriá, J.M. \& Doblas, M. (2002). Cenozoic volcanism I: the Iberian Peninsula. In: The Geology of Spain (Gibbons, W. \& Moreno, T. Eds.). The Geological Society, London, 417-438.

Maaløe, S. (1973). Temperature asd pressure relations of ascending primary magmas. Journal of Geophysical Research, 78: 6877-6886. http://dx.doi.org/10.1029/ JB078i029p06877.

Martí, J.; Planagumà, L.; Geyer, A.; Canal, E. \& Pedrazzi, D. (2011). Complex interaction between Strombolian and phreatomagmatic eruptions in the Quaternary monogenetic volcanism of the Catalan Volcanic Zone (NE of Spain). Journal of Volcanology and Geothermal Research, 201: 178-193. http://dx.doi. org/10.1016/j.jvolgeores.2010.12.009.

Meschede, M. (1986). A method of dicriminating between different types of mid-ocean ridge basalts and continental tholeiites with the $\mathrm{Nb}-\mathrm{Zr}-\mathrm{Y}$ diagram. Chemical Geology, 56: 207-218. http://dx.doi. org/10.1016/0009-2541(86)90004-5.

Nicholson, H. \& Latin, D. (1992). Olivine tholeiites from Krafla, Iceland: evidence for variation in melt fraction within a plume. Journal of Petrology, 33: 1105-1024. http://dx.doi.org/10.1093/petrology/ 33.5.1105. 
Pearce, J.A. \& Cann, J.R. (1973). Tectonic setting of basic volcanie rocks determined using trace element analysis. Earth and Planetary Science Letters, 19:290-300. http://dx.doi.org/10.1016/0012-821X(73)90129-5.

Porrit, L.A.; Russell, J.K. \& Quane, S.L. (2012). Pele's tears and spheres: Examples from Kilauea Iki. Earth and Planetary Science Letters, 333-334: 171-180. http://dx.doi.org/10.1016/j.eps1.2012.03.031.

Ramírez Merino, J.I.; Ancoechea, E. \& Pérez González, A. (1985). Mapa Geológico de España 1:50.000, hoja $\mathrm{n}^{\circ} 785$ (Almagro). IGME.

Stovall, W.K.; Houghton, B.F.; Gonnermann, H.M.; Fagents, S.A. \& Swanson, D.A. (2010). Eruption dynamics of Hawaiian-style fountains: the case study of episode 1 of the Kilauea Iki 1959 eruption. Bulletin of Volcanology, 73: 511-529. http://dx.doi. org/10.1007/s00445-010-0426-z.

Sumner, J.M. (1998). Formation of clastogenica lava flows during fissure eruption and scoria cone collapse: the 1986 eruption of Izu-Oshima volcano, eastern Japan. Bulletin of Volcanology, 60: 195-212. http://dx.doi.org/10.1007/s004450050227.

Sun, S.S. \& McDonough, W.F. (1989). Chemical and isotopic systematics of oceanic basalts: implications for mantle composition and processes. In: Magmatism in the Ocean Basins (Saunders, A.D. \& Norry, M.J. eds.). Geological Society, London, Special Publication 42: 313-345.

Treuil, M. \& Joron, J. M. (1975). Utilisation des éléments hygromagmatophiles pour la simplification de la modélisation quantitative des processus magmatiques. Exemples de l'Afar et de la dorsade médioatlantique. Société Italiana Mineralogié et Petrologié, 31: 125-प००.

Valentine, G.A.; Perry, F.V. \& Wolde, G. (2000). Field characteristics of deposits from spatter-rich pyroclastic density currents at Summer Coon volcano, Colorado. Journal of Volcanology and Geothermal Research, 104: 187-199. http://dx.doi.org/10.1016/ S0377-0273(00)00206-7.

Valentine, G.A.; Perry, F.; Krier, D.; Keating, G.N.; Kelley, R.E. \& Cogbill, A.H. (2006). Small volume basaltic volcanoes: eruptive products and processes and posteruptive geomorphic evolution in Crater Flat (Pleistocene), southern Nevada. GSA Bulletin, 118, 1313-1330. http://dx.doi.org/10.1130/B25956.1.

Weaver, B.L. (1991). Trace element evidence for the origin of ocean-island basalts. Geology, 19: 123-126. http:// dx.doi.org/10.1130/0091-7613(1991)019<0123: TEEFTO $>2.3 . \mathrm{CO} ; 2$.

Wilson, M. (ed.) (1989). Igneous Petrogenesis. Unwin Hyman Ltd, London, 466 pp. http://dx.doi.org/10. 1007/978-1-4020-6788-4.

Wood, D.A. (1980). The application of a Th-Hf-Ta diagram to problems of tectonomagmatic classification and to establishing the nature of crustal contamination of basaltic lavas of the British Tertiary volcanic province. Earth and Planetary Science Letters, 50: 11-30. http://dx.doi.org/10.1016/0012-821X(80)90116-8. 